\title{
Timing of Locomotor Recovery from Anoxia Modulated by the white Gene in Drosophila
}

\author{
Chengfeng Xiao and R. Meldrum Robertson ${ }^{1}$ \\ Department of Biology, Queen's University, Kingston, Canada K7L 3N6 \\ ORCID IDs: 0000-0002-2301-1365 (C.X.); 0000-0002-2666-7861 (R.M.R.)
}

\begin{abstract}
Locomotor recovery from anoxia follows the restoration of disordered ion distributions and neuronal excitability. The time taken for locomotor recovery after $30 \mathrm{sec}$ anoxia (around $10 \mathrm{~min}$ ) is longer than the time for the propagation of action potentials to be restored $(<1 \mathrm{~min})$ in Drosophila wild type. We report here that the white $(w)$ gene modulates the timing of locomotor recovery. Wildtype flies displayed fast and consistent recovery of locomotion from anoxia, whereas mutants of $w$ showed significantly delayed and more variable recovery. Genetic analysis including serial backcrossing revealed a strong association between the $w$ locus and the timing of locomotor recovery, and haplo-insufficient function of $w^{+}$in promoting fast recovery. The locomotor recovery phenotype was independent of classic eye pigmentation, although both are associated with the $w$ gene. Introducing up to four copies of mini-white $\left(\mathrm{mw} \mathrm{w}^{+}\right)$into $\mathrm{w} 1118$ was insufficient to promote fast and consistent locomotor recovery. However, flies carrying $\mathrm{w}^{+}$duplicated to the $Y$ chromosome showed wild-type-like fast locomotor recovery. Furthermore, Knockdown of $w$ by RNA interference (RNAi) in neurons but not glia delayed locomotor recovery, and specifically, knockdown of $w$ in subsets of serotonin neurons was sufficient to delay the locomotor recovery. These data reveal an additional role for $w$ in modulating the timing of locomotor recovery from anoxia.
\end{abstract}

KEYWORDS Drosophila; white gene; anoxia; locomotor recovery; serotonin neuron

DULT Drosophila enter a coma-like state during exposure to anoxia. The changes due to anoxia in Drosophila photoreceptors and the mammalian CNS include the occurrence of anoxic depolarization, disordered ion distributions, and the loss of neuronal excitability (Hansen 1985; Agam et al. 2000; Takano et al. 2007). On returning to normoxia, the timing of recovery varies depending on specific processes. Recovery of neural $\mathrm{K}^{+}$distribution after $<30 \mathrm{sec}$ anoxia starts immediately and extracellular $\mathrm{K}^{+}$concentration is almost back to normal within $30 \mathrm{sec}$ (Armstrong et al. 2011). Propagation of action potentials recovers within 1 min whereas complex motor activity, such as continuous walking, takes longer (around $10 \mathrm{~min}$ ) to recover (Krishnan et al. 1997). Thus the recovery of locomotor coordination involves not only the re-establishment of intercellular communication between neurons, and from neuron to muscle, but also the return of appropriate synaptic strength and coordination. Factors

Copyright @ 2016 by the Genetics Society of America doi: 10.1534/genetics.115.185066

Manuscript received November 20, 2015; accepted for publication March 26, 2016; published Early Online March 29, 2016.

${ }^{1}$ Corresponding author: 3118 Biosciences Complex, Department of Biology, Queen's University, Kingston, Canada K7L 3N6. E-mail: robertrm@queensu.ca influencing ion channels, membrane fluidity, and vesicle recycling, together with the coordination of a variety of modulatory signaling cascades, could therefore have considerable impact on locomotor recovery. The recovery process is complicated and so far little of the cellular or molecular basis regulating the timing of locomotor recovery from anoxia is understood.

The white $(w)$ gene, discovered by Thomas Hunt Morgan (1910), encodes a subunit of a heterodimer transmembrane protein that functions as an ATP-binding cassette transporter (O'Hare et al. 1984; Hazelrigg 1987). The White protein is involved in the uptake of precursors into granules for the synthesis of pteridine (red) and ommochrome (brown) pigments (O'Hare et al. 1984; Dreesen et al. 1988; Tearle et al. 1989). In the Malpighian tubule, the $w^{+}$transport system is associated with the accumulation of intracellular 3-hydroxykynurenine and cyclic guanosine monophosphate (cGMP) in the granules/vesicles (Sullivan and Sullivan 1975; Evans et al. 2008). The $w^{+}$transport system also transports the pigment precursors, guanine and tryptophan, crossing cytoplasmic membranes to contribute to pigment synthesis (Green 1949; Sullivan et al. 1979; Sullivan et al. 1980; Ferré et al. 1986). Many other substrates, including biogenic amines (dopamine, serotonin, and histamine), metabolic intermediates, and small molecules are transported by this 
system (Anaka et al. 2008). Levels of dopamine and serotonin, important modulators of synaptic transmission, are reduced in the heads of $w$ mutants relative to the wild type according to some but not all reports (Borycz et al. 2008; Sitaraman et al. 2008; Simon et al. 2009). Mutations at the $w$ locus, including complete lack of transcription in null mutants, altered amounts of transcript, and mislocation to neighboring heterochromatin, are associated with impaired pigment transport and deposition in the compound eyes (Nolte 1950; Pirrotta and Bröckl 1984). In addition to eye pigmentation, extraretinal functions of $w$ have been observed (Zhang and Odenwald 1995; Campbell and Nash 2001). In the context of locomotor recovery from anoxia, such a $w^{+}$transport system could have critical housekeeping functions in building up vesicle stores, promoting synchronous release and recycling of small molecules, and facilitating fast recovery of ion disorders and neural transmission.

By characterizing the dynamics of locomotion before, during, and after a transient anoxia, we found that white-eyed mutant w1118 flies took longer to recover locomotion and were more variable compared with wild-type Canton-S (CS). The w1118 strain carries a null mutant allele of $w$ in the X chromosome, and is heavily used as genetic background for generating transgenic lines. Both w1118 and CS flies are common controls for genetic study in Drosophila. In this report we provide evidence of an association between the $w$ allele and locomotor recovery. Our data, collected using multiple approaches including serial backcrossing, RNA interference (RNAi) knockdown, and $w^{+}$duplication to Y chromosome, strongly support a conclusion that $w^{+}$modulates the timing of locomotor recovery from anoxia.

\section{Materials and Methods}

\section{Fly strains}

Fly strains used for the experiments were wild-type CS (\#1, Bloomington stock center); w1118 (L. Seroude laboratory); additional wild types including Hikone-AS (\#3), Amherst-3 (\#4265), Florida-9 (\#2374); and mutants including $w^{1}$ (\#145), $w^{a}$ (\#148), and $w^{c f}$ (\#4450). The w1118 was reisogenized using a balancer stock $w^{1118} ; \mathrm{T}(2 ; 3) \mathrm{ss}^{\mathrm{aD}} / \mathrm{CyO} ; \mathrm{TM} 3$, $\mathrm{Sb}$ (L. Seroude laboratory) by following a reported protocol (Garfinkel et al. 2004). The other fly strains and their sources were: duplication line Raf ${ }^{11} / \mathrm{FM} 6,1(1) \mathrm{FMa}^{1} / \mathrm{Dp}(1$; Y)B $\mathrm{B}^{+} w^{+} y^{+}$(\#5733), elav-Gal4 (\#8765), repo-Gal4 (\#7415), UAS-w-RNAi (1) (\#31088) and UAS-w-RNAi (2) (\#35573), $y w \Delta \Delta 1 ; c n, b w$ (L. Seroude laboratory), Janelia UAS lines pJFRC2 (\#32189), pJFRC5 (\#32192), and pJFRC7 (\#32194) (Pfeiffer et al. 2010), UAS-hsp70 (Xiao et al. 2007), UAS-Httex1Qn-eGFP ( $n=47$ or 103) (Zhang et al. 2010); UAS-hsp26 and UAS-hsp27 (Wang et al. 2004), UAS-w-eYFP (Evans et al. 2008), R50E07-, R50H05- and R50E11-Gal4 (Pfeiffer et al. 2008). $w^{+}$; $c n, b w$ were generated according to standard genetic methods in Drosophila (Greenspan 2004). The pJFRC2-7 flies were backcrossed into newly isogenized w1118 according to previous protocol (Garfinkel et al. 2004). UAS-w-RNAi flies were backcrossed to CS background by a two-step procedure. Male RNAi flies were first crossed into $w^{+} ;$;TM3/TM6, and male progeny $\left(w^{+} / \mathrm{y} ; ; \mathrm{UAS}-w\right.$-RNAi/TM3) were backcrossed into $w^{+} ;$;TM3/TM6 for establishing $w^{+} ;$;UAS- $w$-RNAi stock.

Flies were maintained on standard cornmeal medium at room temperature $\left(21-23^{\circ}\right)$ in $12 \mathrm{hr} / 12 \mathrm{hr}$ of light/dark illumination with light on at $7 \mathrm{Am}$ and off at $7 \mathrm{PM}$. Adult flies were collected within two days after emergence, transferred into fresh food vials and subjected to locomotor assay at least 3 days after collection. A period of 3 days free of anoxic exposure was guaranteed before the test. Experiments were performed during the daytime between $10 \mathrm{Am}$ and $4 \mathrm{PM}$ to avoid the morning and evening locomotor peaks (Stoleru et al. 2004).

\section{An apparatus for locomotor assay}

An apparatus was used for the locomotor assay of multiple flies at the same time (Xiao and Robertson 2015). Briefly, circular arenas $(1.27 \mathrm{~cm}$ diameter) were drilled in a $0.3-\mathrm{cm}$-thick Plexiglas plate. The $0.3-\mathrm{cm}$ thickness allowed flies to turn around but prevented vertical movement, thus the twodimensional videos represented locomotion constrained to the horizontal plane. The bottom surface of the arenas was covered with chromatography paper (Cat\# 05-714-4, Fisher Scientific, Pittsburgh, PA) for air circulation. The top was covered with another sliding and slightly larger Plexiglas sheet with holes $(0.3-\mathrm{cm}$ diameter) close to one end for fly loading. The plate was then positioned in a larger container designed for nitrogen exposure. The plate was illuminated with a white light box (Logan Portaview slide/transparency viewer). White cardboard screens were used for light reflection to improve illumination from the sides, and to shield the arenas from light in the room. Locomotor activities of flies were recorded with a video camera (Logitech Webcam C905) and its associated software. Grayscale videos, formatted as Windows Media Video (WMV) at a frame rate of $15 \mathrm{fps}$ and a resolution of $1600 \times 1200$ pixels, were taken and stored for post hoc analysis. Experimental settings, including illumination, light reflection, and camera configuration, remained consistent from experiment to experiment.

\section{Analysis of locomotor recovery from anoxia}

Locomotor activities before, during, and after a short period of anoxia (pure nitrogen exposure at a speed of 10 liter/min) were analyzed. Flies no older than 9 days were exposed to a 30 sec anoxia after 5 min of pre-anoxia locomotion and then observed for $1 \mathrm{hr}$ during recovery under normoxia. A slow air flow (2 liter/min) was provided throughout the experiment, except for the period of $30 \mathrm{sec}$ nitrogen exposure, to remove the effect of dead space. A prior acclimatization period ( $5 \mathrm{~min}$ ) was allotted before the experiment to avoid initial elevated activities (Liu et al. 2007). We chose a $30 \mathrm{sec}$ nitrogen pulse because male flies usually entered anoxic coma around 10$15 \mathrm{sec}$ from the onset of anoxia in our experimental settings, and $30 \mathrm{sec}$ exposure was sufficient to knockdown all flies without exception. During recovery, most of the mutants 
started to walk continuously within 30 min, whereas a few mutants required a longer time to walk. We therefore recorded $1 \mathrm{hr}$ of recovery after anoxia. Preliminary experiments showed that, although the flies started to walk within $1 \mathrm{hr}$, normal locomotor activities were not completely recovered to the nonexposed control level in w1118 flies. After 1 day, recovery from the $30 \mathrm{sec}$ anoxia is likely complete (unpublished observations). Thus a period of 3 days free of anoxic exposure was guaranteed before experiments to avoid effects of anesthetization during fly collection.

\section{Fly tracking}

Fly tracking was performed according to the protocol reported previously (Xiao and Robertson 2015). Briefly, a script was developed to track fly position using free software Open Computer Vision 2.0 (OpenCV2.0) operating in the environment of Microsoft Visual C ++2008 Express (Redmond, WA). The path length per second was calculated every $0.2 \mathrm{sec}$. The main scripting procedures included: (1) learning a specific background from multiple frames of a video, (2) comparing the difference between each frame and the background, (3) computing the center of mass of each fly, and (4) calculating path length per second. A metric ruler was placed in the camera view alongside the arenas for pixel-mm conversion.

\section{Evaluation of time to locomotor recovery (TR)}

Wild-type flies were not ready to walk continuously until around $10 \mathrm{~min}$ after $30 \mathrm{sec}$ anoxic exposure. There were variations for the time to start locomotion from fly to fly and from strain to strain. A threshold criterion was established to evaluate the time required to walk continuously, which we termed as locomotor recovery. Definition of a threshold was based on 5 min locomotion before anoxia. In a pretest with w1118 flies, the $25^{\text {th }}$ percentile of 300 values of path length per second (equivalent to $5 \mathrm{~min}$ locomotion) was around $0.3 \mathrm{~cm}$. We thus used $0.3 \mathrm{~cm}$ of path length per second as the threshold for determining locomotor recovery. Additionally, to avoid taking into account early sporadic events (e.g., wing closure or body roll-over) that could result occasionally in above-threshold path lengths, a criterion of at least 10 instances of path length per second at or above threshold $(0.3 \mathrm{~cm} / \mathrm{sec})$ within 60 consecutive seconds was set as the standard for recovered locomotion. The TR was measured as the earliest time to meet this criterion.

\section{Immunohistochemistry}

Experimental procedures were based on two protocols $(\mathrm{Wu}$ and Luo 2006; Jenett et al. 2012) with minor modification. Briefly, dissected brain tissues were fixed in freshly prepared 4\% paraformaldehyde for $1 \mathrm{hr}$. After three washes with PAT (PBS with $0.5 \%$ BSA and 0.5\% Triton X-100), tissues were incubated with blocking buffer (10\% goat serum in PAT) at room temperature for $1 \mathrm{hr}$. Brain tissues were then incubated with primary antibodies: mouse anti-GFP supernatant (12A6, Developmental Studies Hybridoma Bank) at 1:20, rat antiserotonin (YC5/45, Accurate Chemical and Scientific Corporation) at 1:20, in blocking buffer for 1-2 days. Following three washes, tissues were incubated with Alexa Fluor 488conjugated goat anti-mouse IgG (115-545-003, Jackson ImmunoResearch) at 1:500 and Cy3-conjugated goat antirat IgG (112-165-003, Jackson ImmunoResearch). Secondary incubation was performed in the dark for 1-2 days. After three washes, tissues were suspended in $200 \mu$ l SlowFade Gold antifade reagent (S36938, Life Technologies) and mounted on slides for microscopy. Image stacks were taken using a Carl Zeiss LSM 710 NLO laser scanning confocal/ multiphoton microscope and processed with associated software Zen 2009 (Carl Zeiss, Thornwood, NY).

\section{Statistics}

Because part of the data showed non-Gaussian distribution, nonparametric tests (Mann-Whitney $U$ test or Kruskal-Wallis test with Dunn's multiple comparison) were performed to compare the difference of medians between two or multiple groups. TR was evaluated from eight flies $(n=8)$ for each test. More than three duplications of locomotor assays were conducted. Specific statistical methods are provided in the text or figure legends where applicable.

\section{Data availability}

The authors state that all data necessary for confirming the conclusions presented in the article are represented fully within the article.

\section{Results}

\section{Delayed locomotor recovery from anoxia in w1118 strain}

During recovery from anoxia flies performed sequential actions in the following order: rhythmic leg twitching, wing closure, rolling over, standing upright, antennal grooming, and walking. Most of the recovery time occurs during the return to normoxia to rhythmic twitching of legs, and from standing upright to walking. Wild-type flies do not walk continuously until around $10 \mathrm{~min}$ after a $30 \mathrm{sec}$ anoxic exposure. We examined locomotor activity in adult flies by computing path length per second before and after an anoxia using an assay described previously (Xiao and Robertson 2015). CS and w1118 male flies walked continuously in small circular arenas (1.27-cm diameter) under normoxia. A 30 sec anoxia was sufficient to induce coma in all flies after which locomotor activity gradually recovered (Figure 1, A and B). CS male flies recovered with a median of $530.0 \mathrm{sec}$ [interquartile range (IQR) 461.8-553.0 sec, $n=8$ ], whereas locomotor activity of w1118 males recovered at a median of $1037.0 \mathrm{sec}$ (IQR 755.3-1797.0 sec, $n=8$ ). The w1118 males displayed longer TR than CS males $(P<0.05$, $t$-test with Welch's correction), and larger variance of TR than CS males $(P<0.0001, F$-test) (Figure 1C).

\section{Mutants of $w$ showed delayed locomotor recovery from anoxia}

To determine whether the $w$ mutation could be involved in the delayed locomotor recovery from anoxia, several 
A

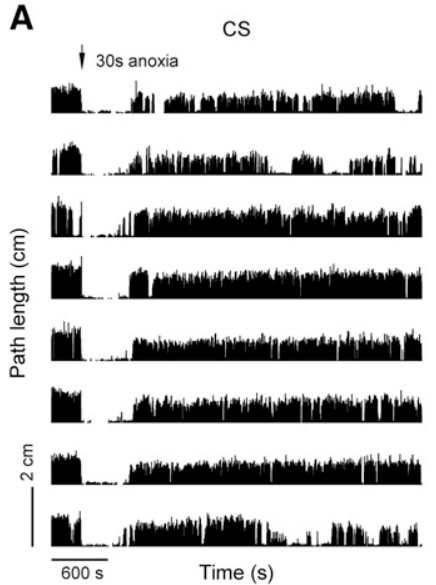

B

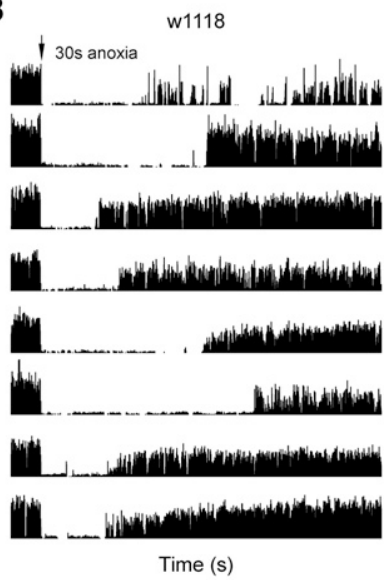

C

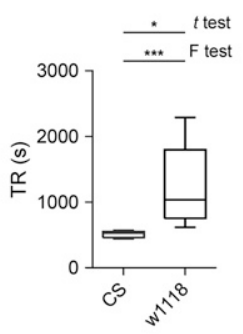

D

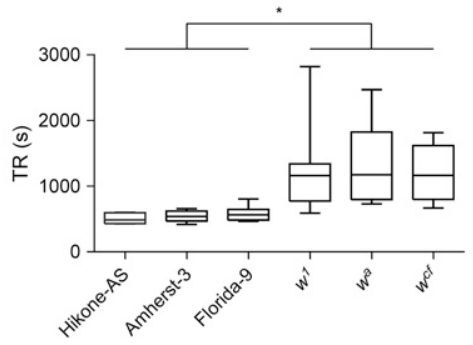

Figure 1 The $w$ mutants display delayed locomotor recovery from anoxia. (A) Locomotor activity of CS flies in the circular arenas $(1.27-\mathrm{cm}$ diameter and $0.3-\mathrm{cm}$ depth). Each trace represents $1 \mathrm{hr}$ locomotion of a single fly. The path length per second vs. time are plotted. A $30 \mathrm{sec}$ anoxia (arrow head) is applied during 300-330 sec. Flies are otherwise provided with normal air flow (at 2 liter/min). (B) Locomotor activity of w1118 flies in the same arenas and subjected to the same treatment as CS. (C) Analysis of TR in CS and w1118 flies. *P<0.05 (t-test), $* * * P<$ 0.001 ( $F$-test). (D) TR analysis in wild types (Hikone-AS, Amherst-3, and Florida-9) and mutants $\left(w^{1}, w^{a}\right.$, and $\left.w^{c f}\right)$. $* P<0.05$ by Kruskal-Wallis test.

additional wild-type strains including Hikone-AS, Amherst-3, and Florida-9 captured in different locations and dates, as well as the $w$ mutants including $w^{1}$ (O'Hare et al. 1984), $w^{a}$ (Gehring and Paro 1980; Bingham and Judd 1981; Pirrotta and Bröckl 1984) and $w^{c f}$ (Mackenzie et al. 1999) were examined. The TRs in Hikone-AS (median $485.0 \mathrm{sec}$, IQR 429.8-593.8 sec, $n=$ 8), Amherst-3 (median $538.5 \mathrm{sec}$, IQR 466.5-621.0 sec, $n=8$ ), and Florida-9 (median $562.5 \mathrm{sec}$, IQR 483.0-646.5 sec, $n=8$ ) were comparable with no significant difference. The TRs in $w^{1}$ (median $1162.0 \mathrm{sec}$, IQR 772.5-1338.0 sec, $n=12$ ), $w^{a}$ (median $1173.0 \mathrm{sec}$, IQR 795.0-1822.0 sec, $n=15$ ), and $w^{c f}$ (median $1166.0 \mathrm{sec}$, IQR 797.0-1617.0 sec, $n=8$ ) were similar with no statistically significant difference. The $w$ mutants displayed prolonged TRs compared with Hikone-AS $(P<0.05$ for all three comparisons, Kruskal-Wallis test with Dunn's multiple comparison), Amherst-3 ( $P<0.05$, Kruskal-Wallis test with Dunn's multiple comparison), or Florida-9 $(P<0.05$, Kruskal-Wallis test with Dunn's multiple comparison) (Figure 1D). Thus, the $w$ mutants showed delayed locomotor recovery from anoxia compared with wild types.

\section{$w^{+}$allele was associated with fast locomotor recovery from anoxia}

The w1118 strain carries isogenic $\mathrm{X}$, second and third chromosomes and a null allele $w^{1118}$ on the $\mathrm{X}$ chromosome (Pirrotta and Bröckl 1984; Levis et al. 1985a). w1118 differs from CS in two main genetic components: the $w$ allele and genetic background. Fast locomotor recovery from anoxia in wild type might be associated with the $w^{+}$allele or the genetic background excluding the $w$ locus. We next explored the genetic contribution of the $w^{+}$allele or genetic background to the timing of locomotor recovery.

By crossing male w1118 to female CS, and reciprocally, male CS to female w1118, two F1 male progenies were generated: $w^{+} / \mathrm{y}(\mathrm{F} 1)$ and $w^{1118} / \mathrm{y}(\mathrm{F} 1)$, which carried different $\mathrm{X}$ chromosomes and the same heterozygous genetic background on the second and third chromosomes. The median TR in $w^{+} / \mathrm{y}$ (F1) flies was $424.0 \mathrm{sec}$ (IQR 411.5-428.0 sec, $n=8$ ), whereas the median TR in $w^{1118} / \mathrm{y}$ (F1) flies was $662.5 \mathrm{sec}$ (IQR 613.0-786.5 sec, $n=8$ ). Locomotor recovery in $w^{+} / \mathrm{y}$ (F1) flies was faster compared with $w^{1118} / \mathrm{y}$ (F1) flies $(P<0.05$, Mann-Whitney $U$-test) (Figure $2 \mathrm{~A})$.

To examine the contribution of wild-type genetic background, introgression (Kain et al. 2012) was performed to gradually replace isogenic second and third chromosomes in w1118 with wild-type counterparts. Briefly, by crossing male CS to female w1118, the heterozygous female progeny (F1) was collected and backcrossed to CS males. The backcross was conducted for three consecutive generations. The resulting $w^{+} / \mathrm{y}(\mathrm{F} 4)$ and $w^{1118} / \mathrm{y}(\mathrm{F} 4)$ flies contained synchronized and mostly wild-type genetic background. The median TRs were $525.5 \mathrm{sec}$ (IQR 509.0-554.5 sec, $n=8$ ) in $w^{+} / \mathrm{y}$ (F4) flies, and $797.5 \mathrm{sec}$ (IQR 512.3-1032.0 sec, $n=8$ ) in $w^{1118} / \mathrm{y}$ (F4) flies. The TR was shorter in $w^{+} / \mathrm{y}$ (F4) flies than $w^{1118} / \mathrm{y}$ (F4) flies $(P<0.05$, Mann-Whitney $U$-test) (Figure 2B). Hence, fast locomotor recovery was tightly associated with $w^{+}$-carrying flies.

To further examine the association between $w^{+}$allele and fast locomotor recovery, serial backcrossing was conducted to exchange $w$ alleles between CS and w1118. Initially, male CS was crossed to female w1118, and reciprocally, male w1118 was crossed to female CS. Heterozygous female progeny (F1) was backcrossed with w1118 or CS respectively. The backcrossing was performed for nine more generations. The $w^{+} / \mathrm{y}(\mathrm{F} 10)$ and $w^{1118} / \mathrm{y}(\mathrm{F} 10)$ flies were collected and examined. The median TRs were $556.0 \mathrm{sec}$ (IQR 499.8-645.3 sec, $n=8$ ) in $w^{+} / \mathrm{y}(\mathrm{F} 10)$, and 793.5 sec (IQR 673.3-1439.0 sec, $n=8)$ in $w^{1118} / \mathrm{y}(\mathrm{F} 10)$. The TR was longer in $w^{1118} / \mathrm{y}$ (F10) flies than $w^{+} / \mathrm{y}(\mathrm{F} 10)$ flies $(P<0.05$, Mann-Whitney $U$-test) (Figure 2C). Thus the $w^{+}$allele was strongly associated with fast locomotor recovery from anoxia.

\section{Flies with $w^{+}$duplicated to $Y$ chromosome displayed fast locomotor recovery}

To further support the association between $w$ allele and the phenotype of locomotor recovery, we examined TR in flies 
A

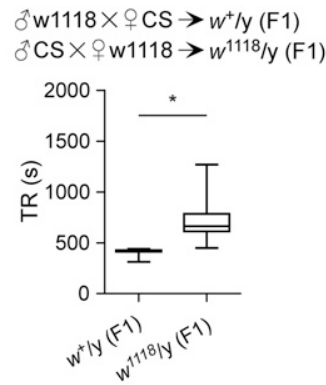

B

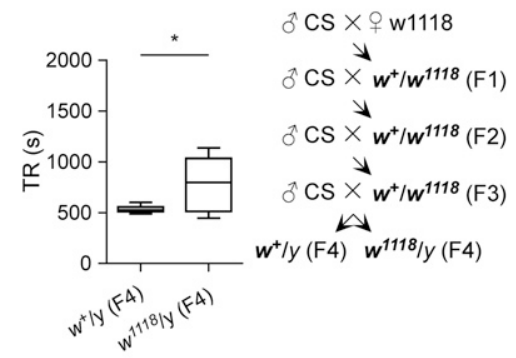

C
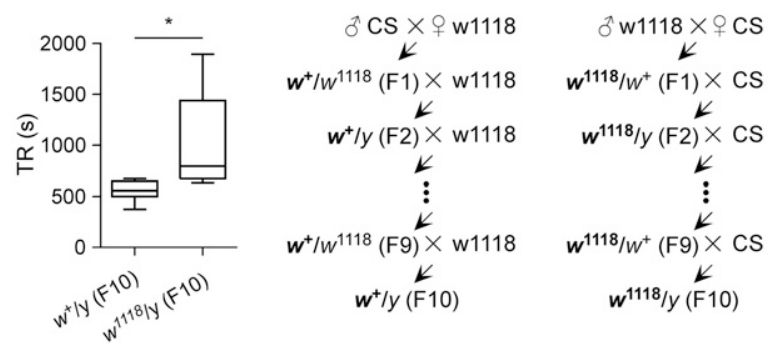

D

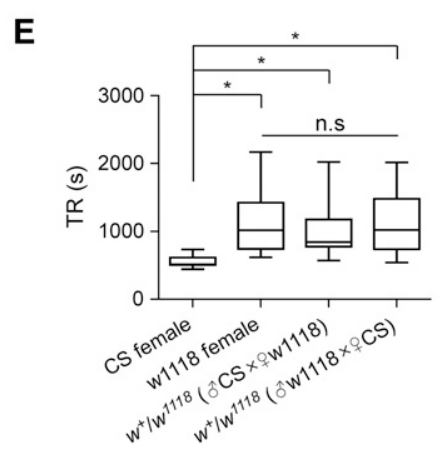

Figure $2 W^{1118}$ allele is associated with delayed locomotor recovery from anoxia. (A) TR analysis in the progeny flies from the cross between CS and w1118. The specific crossed are indicated. $* P<0.05$ by Mann-Whitney U-test. (B) TR analysis in flies generated by introgression (see Materials and Methods). * $P<0.05$ by Mann-Whitney U-test. (C) TR analysis in flies generated by serial backcrossing. * $P<0.05$ by Mann-Whitney $U$-test. (D) TR analysis in CS and flies carrying a $w^{+}$duplicated to $Y$ chromosome. (E) TR analysis in these female flies: CS, $W 1118, w^{+} / w^{1118}$ (progeny of male CS and female $w 1118$ ) and $w^{+} / w^{1118}$ (progeny of male $w 1118$ and female CS). $* P<0.05$ by Kruskal-Wallis test; n.s, nonsignificant difference.

carrying $w^{+}$duplicated to the $\mathrm{Y}$ chromosome. The TR in $w^{1118} / \mathrm{Dp}\left(1 ; \mathrm{Y} \mathrm{B}^{\mathrm{S}} w^{+} y^{+}\right.$(median $523.0 \mathrm{sec}$, IQR 472.8$600.0 \mathrm{sec}$ ) was similar to the TR in CS males (median $517.0 \mathrm{sec}$, IQR $484.3-530.3 \mathrm{sec}$ ) with no significant difference (Figure 2D). Thus, flies carrying $w^{+}$duplicated to the $Y$ chromosome displayed similarly fast locomotor recovery from anoxia as CS.

\section{$w^{+}$allele was haplo-insufficient for fast locomotor recovery in female flies}

We next addressed whether $w^{+}$allele was haplo-sufficient to promote fast locomotor recovery from anoxia in heterozygous female flies. The TR was examined in four different female flies: (1) CS, (2) w1118, (3) $w^{+} / w^{1118}$ from the cross between male CS and female w1118, and (4) $w^{+} / w^{1118}$ from the cross between male w1118 and female CS. The median TR was $514.5 \mathrm{sec}$ (IQR 506.0-615.5 sec, $n=8$ ) for CS females, $1018.0 \mathrm{sec}$ (IQR 745.5-1424.0 sec, $n=8$ ) for w1118 females, $846.0 \mathrm{sec}$ (IQR 776.8-1177.0 sec, $n=8$ ) for $w^{+} /$ $w^{1118}$ females (from male CS to female w1118), and 1022.0 sec (IQR 737.8-1482.0 sec, $n=8$ ) for $w^{+} / w^{1118}$ females (from male w1118 to female CS). The TR in CS females was shorter than that in w1118 females $(P<0.05$, KruskalWallis test with Dunn's multiple comparison), in $w^{+} / w^{1118}$ females (from male CS to female w1118) $(P<0.05$, KruskalWallis test with Dunn's multiple comparison), and in $w^{+} / w^{1118}$ females (from male w1118 to female CS) $(P<0.05$, KruskalWallis test with Dunn's multiple comparison). There was no difference of TR among w1118, $w^{+} / w^{1118}$ (from male CS to female w1118) and $w^{+} / w^{1118}$ (from male w1118 to female CS) (Figure 2E). These results indicate that in females homozygous $w^{+}$alleles were sufficient, whereas heterozygous $w^{+}$allele was insufficient to promote fast locomotor recovery from anoxia.

\section{Independent phenotypes between locomotor recovery from anoxia and eye pigmentation}

The classic function of White protein is to carry and deposit red and brown pigments into the pigment cells of compound eyes, ocelli, Malpighian tubules, and the testis sheath (Nolte 1950; Hazelrigg 1987). We looked for a possible association between phenotypes of locomotor recovery from anoxia and eye pigmentation. Flies carrying cinnabar, brown ( $c n, b w$ ) double mutations in a $w^{+}$background were generated. These flies $\left(w^{+} ; c n, b w\right)$ were white-eyed (Figure 3A) due to complete blockage of both pteridine and ommochrome pigment pathways (Waaler 1921; Ward 1923; Nolte 1954; Ghosh and Forrest 1967; Dreesen et al. 1988). There was no difference of TR between $w^{+}$; cn, bw flies (median $579.0 \mathrm{sec}$, IQR 467.8$674.8 \mathrm{sec}, n=16$ ) and CS (median $494.0 \mathrm{sec}$, IQR 455.0$555.5 \mathrm{sec}, n=16$ ) (Figure 3B). Hence, the phenotype of eye pigment was independent of the timing of locomotor recovery.

To support the dissociation between locomotor recovery and pigmentation phenotype, RNAi knockdown of $w$ was carried out. Eye color and the timing of locomotor recovery in flies with $w$ knockdown were examined. The pan-neuronal driver elav-Gal4 (Armstrong et al. 2011; Dimitroff et al. 2012) was used for targeting RNAi of $w$ to the CNS. There was no visible difference in eye color between $w^{+}$; elav-Gal4/+; UAS- $w$-RNAi/ + flies and controls $\left(w^{+}\right.$; elav-Gal4/+ and $w^{+} ; ;$ UAS- $w$-RNAi/+) (Figure 3C), indicating that $w$ knockdown in the CNS had no visible effect on eye pigmentation. The TR of $w^{+}$; elav-Gal4/+; UAS- $w$-RNAi/ + males was, however, severely delayed compared with controls (Kruskal-Wallis test with Dunn's multiple comparison) (Figure 3D). The RNAi-induced delay of locomotor recovery was confirmed by using two independent RNAi lines targeting different sequences of the $w$ transcript. These results clearly display an independence between phenotypes of locomotor recovery from anoxia and eye pigmentation. 
A

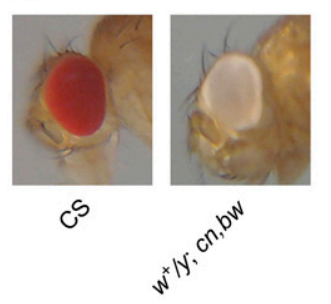

B

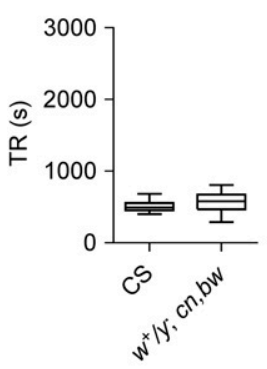

c

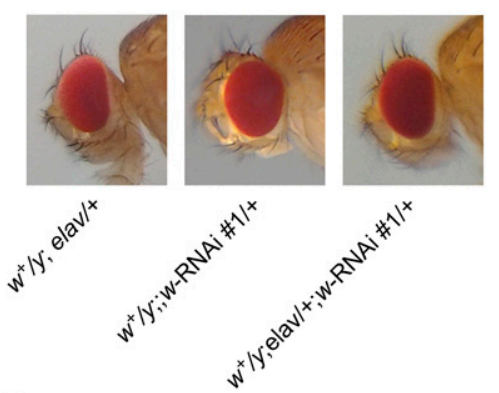

D

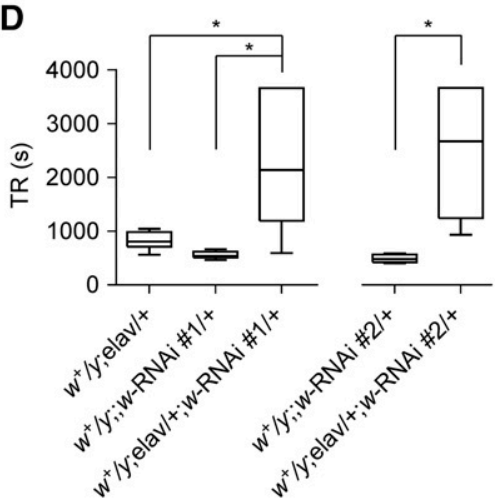

Figure 3 Independent phenotypes between locomotor recovery and eye pigmentation. (A) Eye color in CS and flies carrying cinnabar (cn) and brown (bw) double mutations $\left(w^{+} ; c n, b w\right)$. (B) TR analysis in CS and $w^{+} ; c n, b w .(C)$ Eye color in flies with pan-neuronal $w$-RNAi targeted to the CNS by elav-Gal4 and controls. (D) TR analysis in flies with $w$-RNAi knockdown and their controls. RNAi \#1 and RNAi \#2 indicate two independent RNAi lines targeting different sequences of $w$ mRNA. * $P<$ 0.05 by Kruskal-Wallis test.

\section{mini-white $\left(\mathrm{mw}^{+}\right)$was insufficient to promote fast locomotor recovery}

The $\mathrm{m} w^{+}$contains reduced $5^{\prime}$ and $3^{\prime}$ regulatory sequences and a greatly shortened first intron with a deletion of $\sim 3 \mathrm{~kb}$ HindIIIXbaI fragment (Pirrotta 1988). Depending on its location in the genome, $\mathrm{m} w^{+}$displays a more or less cell-autonomous expression pattern and is controlled by cis-acting regulatory sequences (Hazelrigg et al. 1984; Hazelrigg 1987). In the Drosophila community most transgenic lines carry the $\mathrm{m} \mathrm{w}^{+}$ marker. Because both $w^{+}$and $\mathrm{m} w^{+}$encode the same protein, we examined the contribution of inserted $\mathrm{m} w^{+}$to the timing of locomotor recovery. Importantly, the contribution of $\mathrm{m} w^{+}$, if any, needs to be differentiated from the $w^{+}$allele if the $\mathrm{m} w^{+}$carrying Gal4/UAS expression system (Brand and Perrimon 1993) is introduced into the wild-type genetic background. There were several criteria for these tests: (1) UAS but not Gal4 lines were selected in order to avoid potential modulation by ectopic Gal4 expression (Kramer and Staveley 2003; Rezával et al. 2007), (2) UAS lines with $m w^{+}$-carrying $P$ elements inserted into fixed recombination sites at attP2 or attP40 were preferentially considered in order to minimize the position effect (Nolte 1950; Hazelrigg et al. 1984), and (3) UAS flies carrying multiple copies of $\mathrm{m} w^{+}$were generated to compensate for possibly reduced expression from a single $\mathrm{m} w^{+}$.

We first measured TR in flies carrying the $P$ element (pJFRC2) at attP40 on the second chromosome or the $P$ element (pJFRC2, pJFRC5 or pJFRC7) at attP2 on the third chromosome (Pfeiffer et al. 2010). All the selected UAS lines carry the $\mathrm{m} \mathrm{w}^{+}$marker. They were backcrossed into w1118 for ten generations before experiments by following previously described procedures (Garfinkel et al. 2004). Flies containing multiple copies of $P$ elements were generated by genetic manipulation (Greenspan 2004). We found: (1) TR of each heterozygous UAS line carrying a single $\mathrm{m} w^{+}$was comparable with that of w1118 with no significant differences (Kruskal-Wallis test with Dunn's multiple comparison) (Figure 4A), (2) TR of each homozygous UAS line or the combination of UAS containing two $\mathrm{m} \mathrm{w}^{+}$was similar to that of w1118 with no statistically significant differences (KruskalWallis test with Dunn's multiple comparison) (Figure 4B), and (3) TR of each line carrying four copies of $\mathrm{mw}^{+}$was statistically the same as that in w1118 (Kruskal-Wallis test with Dunn's multiple comparison) (Figure 4C). Therefore, up to four copies of $\mathrm{mw}^{+}$at attP40 or attP2 in the w1118 background were insufficient to promote fast locomotor recovery.

Next we examined TR in UAS flies with nearly random locations of $\mathrm{m} w^{+}$on the $\mathrm{X}$, second or third chromosome. We chose several UAS-hsp70 lines with pINDY5-hsp70 inserted into different chromosomes (Xiao et al. 2007), fly lines carrying UAS-Httex1-Qn-eGFP ( $n=47$ or 103) in different chromosomes (Zhang et al. 2010), two different lines of UAS$w$-eYFP (Evans et al. 2008), and a few other UAS flies in our collection. All of the selected UAS flies were generated in the w1118 background, contained $\mathrm{mw}^{+}$marker, and displayed no apparent developmental or morphological abnormalities. TR in each of the fly lines with an $\mathrm{m} w^{+}$copy number ranging from one (Figure 4D), two (Figure 4E) to four (Figure 4F) was similar with that in w1118 with no significant differences (Kruskal-Wallis test with Dunn's multiple comparison). Thus, up to four copies of $\mathrm{m} w^{+}$with nearly random insertions in the genome were insufficient to confer fast locomotor recovery. Notably, the UAS lines with a $P$ element on the $\mathrm{X}$ chromosome (UAS-hsp70/y and UAS-Httex1-Q103-eGFP/y) displayed TR similar to that in w1118, indicating that expression of $\mathrm{mw}^{+}$with potentially stronger dosage compensation (Pirrotta and Bröckl 1984; Qian and Pirrotta 1995) was unable to promote fast locomotor recovery.

\section{Knockdown of $w$ in subsets of serotonin neurons was sufficient to delay locomotor recovery}

We demonstrated above that RNAi knockdown of $w$ using elav-Gal4 severely delayed locomotor recovery (see Figure 3D). These data suggest that White is expressed in CNS neurons. We further examined the possible expression of White in glial cells. RNAi knockdown of $w$ throughout glia using repo-Gal4 (Sepp et al. 2001) with two independent RNAi lines targeting different sequences of $w$ transcript had no effect on TR (Figure 5A). These data indicate no or minor expression of White in glial cells. 

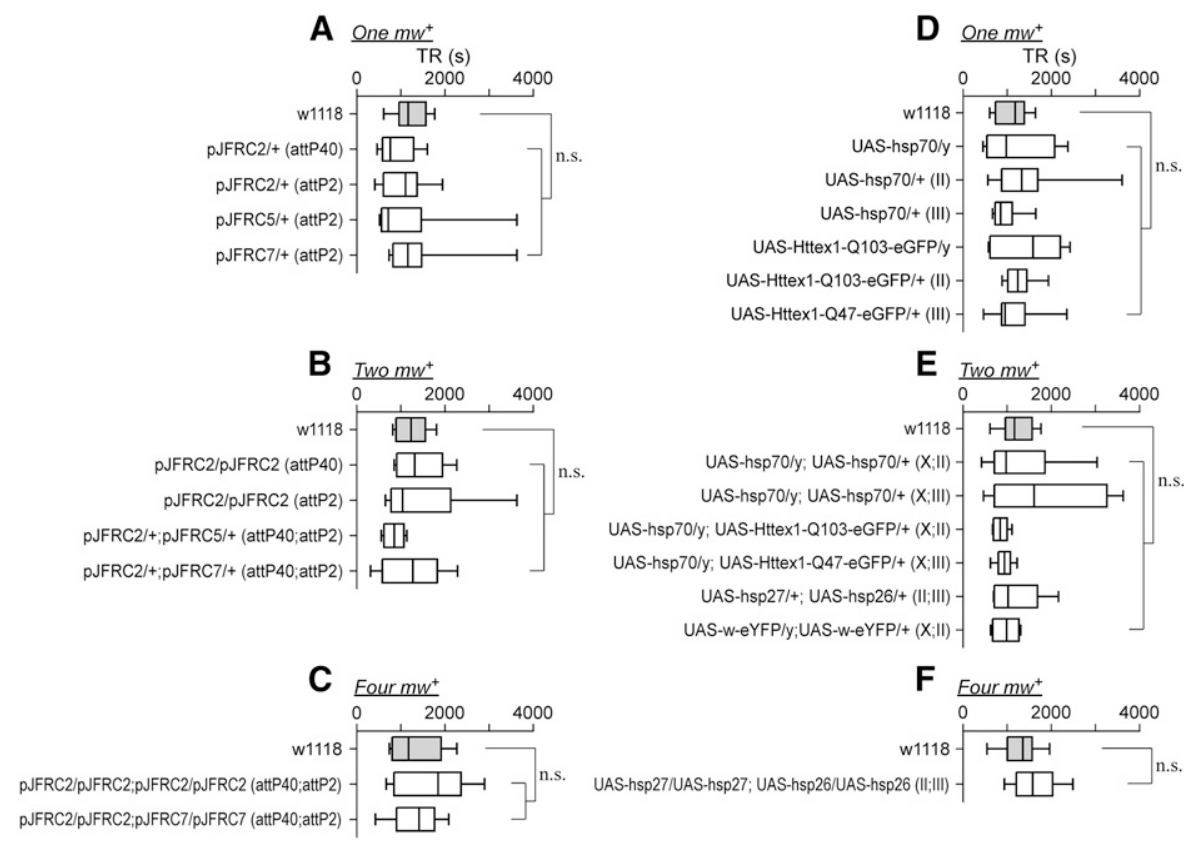

UAS-hsp27/UAS-hsp27; UAS-hsp26/UAS-hsp26 (II;ili) -
Figure 4 The $\mathrm{mw}^{+}$is insufficient to promote fast locomotor recovery. (A-C) TR analysis for flies carrying 1-4 copies of $\mathrm{mw}^{+}$at attP40 or attP2 docking site by site-specific recombination. pJFRC2, pJFRC5 and pJFRC7 are the UAS constructs (Pfeiffer et al. 2010). The specific location of $\mathrm{mw}^{+}$for each fly line is indicated in the parenthesis. n.s, nonsignificant difference. (D-F) TR analysis for flies carrying 1-4 copies of $\mathrm{mw}^{+}$at nearly random locations. The chromosome location for each line is indicated. n.s, nonsignificant difference. Notes: Flies carrying pJFRC2-7 were backcrossed into w1118 for 10 generations before the tests. UAS-hsp70 flies were constructed in the lab and reported previously (Xiao et al. 2007). The other fly lines and sources are: UASHttex1-Qn-eGFP ( $n=47$ or 103) (Zhang et al. 2010); UAS-hsp26 and UAS-hsp27 (Wang et al. 2004); UAS-w-eYFP (Evans et al. 2008).
White is likely expressed in dopaminergic and serotonergic neurons, because a coenzyme tetrahydrobiopterin is required for biosynthesis of both dopamine and serotonin, and tetrahydrobiopterin is an important coproduct in the red pigment pathway that requires White (Ferré et al. 1986). We next performed RNAi knockdown of $w$ using ddc-Gal4 (specific to dopaminergic and serotonergic neurons) and examined the effect on the timing of locomotor recovery. TR in $w^{+} ;$; ddc/ $w$-RNAi flies (median $1298.0 \mathrm{sec}$, IQR $805.0-1608.0 \mathrm{sec}$, $n=8$ ) was clearly prolonged compared with $w^{+} ;$; ddc/+ (median $618.0 \mathrm{sec}$, IQR 593.0-647.5 sec, $n=8$ ) or $w^{+} ; ; w$ $\mathrm{RNAi} /+$ flies (median $616.0 \mathrm{sec}$, IQR 447.3-662.0 sec, $n=8$ ) $(P<0.05$, Kruskal-Wallis test with Dunn's multiple comparisons) (Figure 5B). Results support White expression in dopaminergic and serotonergic neurons and that knockdown of $w$ in these neurons delayed locomotor recovery from anoxia.

Serotonin synthesis begins with hydroxylation of tryptophan, which is also the initial substrate for production of brown pigment xanthommatin, a process requiring White (Sullivan and Sullivan 1975). Serotonin content is greatly reduced in the mutant strain w1118 (Borycz et al. 2008; Sitaraman et al. 2008). We examined whether knockdown of $w$ in serotonin neurons was sufficient to delay locomotor recovery. Three Janelia Gal4 lines, R50E07-, R50H05-, and R50E11-Gal4, each using one of the large intron regions of the serotonin transporter gene (SerT) as enhancers, potentially divide the serotonin population into smaller subsets (Pfeiffer et al. 2008). RNAi knockdown by each of the three Gal4 lines severely delayed locomotor recovery compared with the controls. Delayed locomotor recovery was observed consistently by using two independent RNAi lines (Figure 5C). The expression patterns of R50E07-, R50H05-, and R50E11-Gal4 are illustrated in Figure 5D. Each Gal4 line labeled 20-50 neurons, which were smaller populations than the entire serotonergic family of around 100 neurons (Vallés and White 1988; Giang et al. 2011; Huser et al. 2012). The labeling was consistent with reported patterns (Pfeiffer et al. 2008) and the identity of serotonin subsets was confirmed by double immunostaining using anti-serotonin and anti-GFP antibodies. GFP expression of the R50H05-Gal4 line overlapped with serotonin-positive neurons (Figure 6). Similar results were obtained with R50E07- and R50E11-Gal4 (data not shown). Therefore, knockdown of $w$ in each of the serotonin subsets was sufficient to cause delayed locomotor recovery.

\section{Discussion}

We report here a novel function of $w$ that is tightly associated with the timing of locomotor recovery from anoxia. Downregulation of White, either by mutation or pan-neuronal RNAi knockdown, delayed locomotor recovery and increased its variability. Genetic analysis through serial backcrossing together with simple crossing and introgression confirmed the strong association between $w$ and locomotor recovery phenotype. We further demonstrate the independence of the phenotypes between locomotor recovery and eye pigmentation, although they are both associated with $w$. Moreover, we show that $w^{+}$is haplo-insufficient for fast locomotor recovery in female flies, and that up to four copies of $\mathrm{m} w^{+}$are insufficient to promote fast locomotor recovery. Nevertheless, flies carrying $w^{+}$duplicated to the Y chromosome display wild-type-like fast locomotor recovery. Finally, we show that RNAi knockdown of $w$ in serotonin subsets is sufficient to delay locomotor recovery from anoxia.

\section{The expression pattern of White protein}

White protein is expressed in vesicular but not cytoplasmic membranes in principal cells of Malpighian tubules (Evans 
A

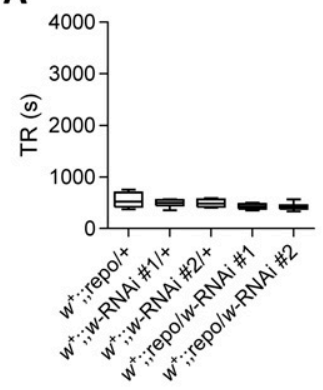

B

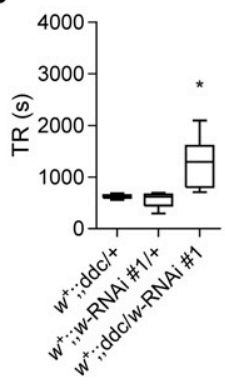

C

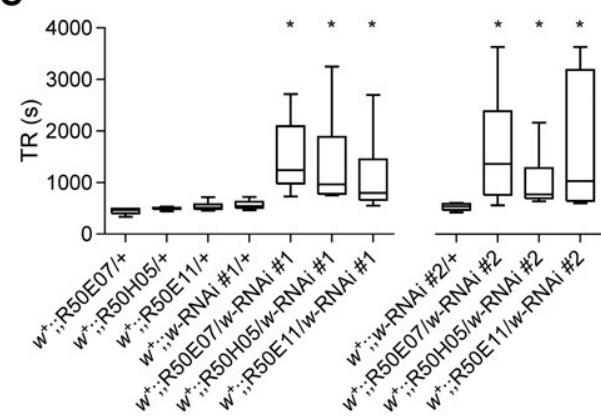

D

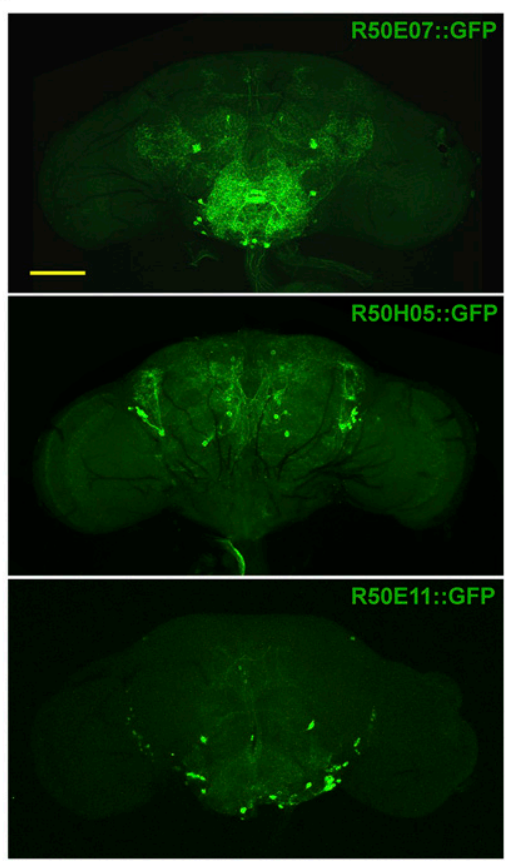

Figure 5 RNAi knockdown of $w$ in serotonin subsets is sufficient to delay locomotor recovery. (A) TR analysis for flies with $w$-RNAi knockdown in pan-glial cells by repo-Gal4. TR was unaffected by $w$-RNAi in pan-glial cells. (B) TR analysis for flies with $w$-RNAi knockdown by ddc-Gal4. Ddc-Gal4 is specific to dopaminergic and serotonergic neurons (Haywood and Staveley 2004). (C) TR analysis for flies with $w$-RNAi knockdown by R5OEO7-, R5OH05- and R5OE11-Gal4. Each of these Gal4 lines uses the enhancers originated from three major introns from SerT (serotonin transporter gene) (Pfeiffer et al. 2008). Two independent RNAi lines (\#1 and \#2) were used for the tests. * $P<0.05$ by Kruskal-Wallis test. (D) GFP expression patterns by R50E07-, R50H05- and R50E11-Gal4. Each line targets 20-50 neurons in the central brain. GFP denotes 20× UASIVS-mCD8::GFP fly line (\#32194). Bar, $100 \mu \mathrm{m}$ for all images. et al. 2008). The $\sim 2.6-\mathrm{kb}$ transcript of $w$ is detectable in the fly head (Pirrotta and Bröckl 1984; Campbell and Nash 2001). Expression of White protein can also be detected in the granules of pigment cells in the lamina and in photoreceptors (Mackenzie et al. 2000; Borycz et al. 2008). However, a convincing expression pattern in the CNS is still lacking. We provide additional evidence for the expression patterns of White protein in the CNS. White is likely expressed in neurons but not glial cells. This is supported by the observations that the delayed locomotor recovery can be induced by panneuronal RNAi knockdown of $w$ or in subsets of serotonin neurons but not in glia.

The level of White in CNS neurons is likely to be extremely low based on the report of unsuccessful immunostaining (Borycz et al. 2008). Meanwhile, the expression is tightly controlled by the adjacent regulatory array of $w$ gene (Hazelrigg et al. 1984; Hazelrigg 1987). Widespread ectopic expression of White from $\mathrm{m} w^{+}$is responsible for male-specific hypersexual behavior (Zhang and Odenwald 1995; Hing and Carlson 1996) and partial recovery of eye color ranging from light yellow to dark red, suggesting that $\mathrm{m} w^{+}$either causes behavioral abnormality, or is insufficient to rescue the loss-of- $w$ function. Consistently, we observed that the insertion of $\mathrm{m} w^{+}$into a mutant background was unable to promote fast locomotor recovery that was typically seen in wild-type flies. It is argued that abnormal expression as well as intracellular mislocation of White in normally expressing cells is associated with male-male courtship behavior (Anaka et al. 2008; Krstic et al. 2013). Hence, $w^{+}$and its expression might represent a highly optimized system in eye pigmentation and substrate transport. Complete lack or downregulation of White could break down such an optimized system and lead to a pro- longed time for restoration of vesicular content during locomotor recovery.

\section{Multiple roles of White protein}

For almost a century White has been known to control eye pigmentation pathways. It was not until 1995 that ectopic expression of White was shown to be associated with malemale courtship behavior (Zhang and Odenwald 1995). Recently, more neural but nonretinal functions of White have been revealed (Hing and Carlson 1996; Campbell and Nash 2001; Borycz et al. 2008; Krstic et al. 2013). In this study, we demonstrate a clear independence of phenotypes between eye pigmentation and the timing of locomotor recovery. We consider below several factors including temporal expression pattern, cell-specificity, and reusability of White-containing granules/vesicles that might explain the potential multiple roles for White.

In the eyes of wild-type flies, White-containing granules carry and deposit two independent eye pigments to the same granules in the pigment cells. The brown pigment is deposited first with a peak being reached only about 1 day after emergence, and then the red pigment is deposited in the same granules for another 4 or 5 days (Nolte 1950). The one-way centrifugal movement of pigment granules and the unchangeable color pattern throughout the life in the mutant $w^{m 4}$ (Nolte 1950) imply that White-containing granules are either not able to return to the cell soma or do so at a very low rate for renewed pigment transport. In the CNS, however, the function of White-containing vesicles could persist. The observation that downregulation of White in neurons delays locomotor recovery in flies up to 9 days old, supports the idea that White continues to function in the adult for $>4$ or 5 days. Also, up to 10-day-old male flies with $\mathrm{mw}^{+}$still display male-male 

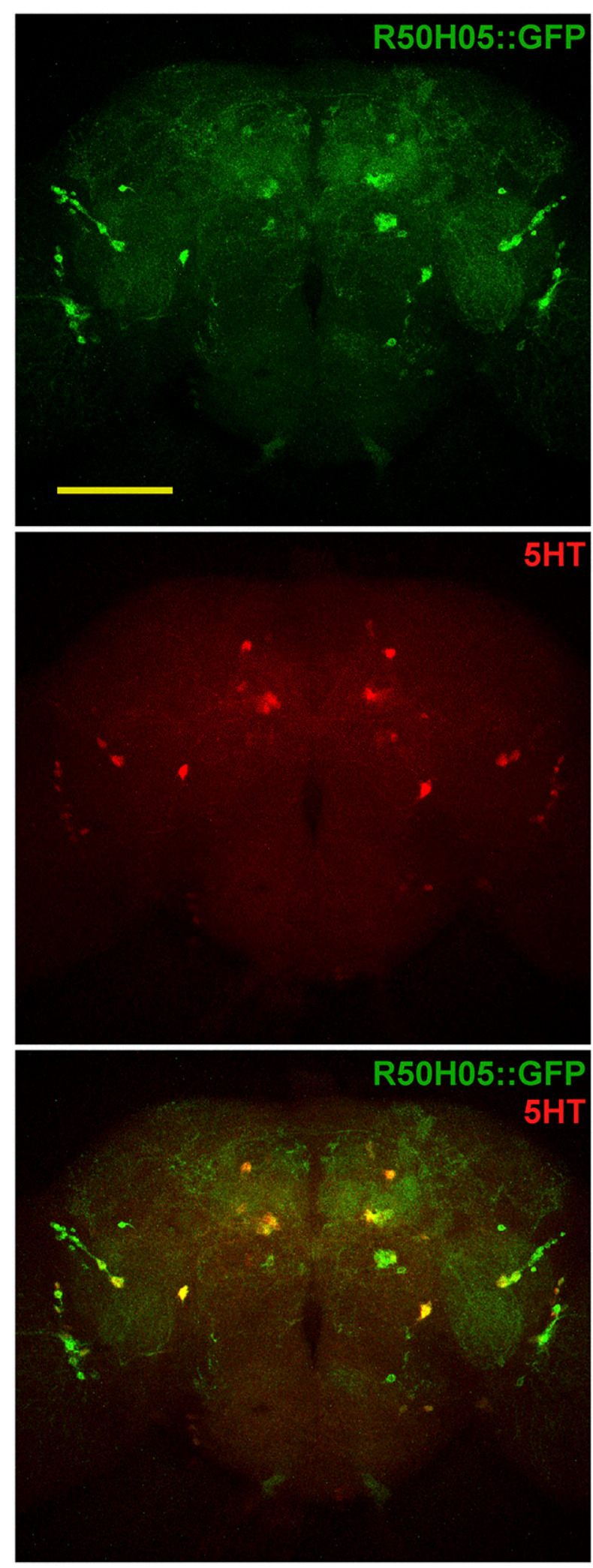

Figure 6 Serotonin identity of R50H05-Gal4 labeled neurons. GFP expression neurons (top panel), serotonin positive neurons (middle panel), and the merge of two images (bottom panel) in the central brain of R50H05::GFP fly. GFP represents 20x UAS-IVS-mCD8::GFP fly line (\#32194). Bar, $100 \mu \mathrm{m}$ for all panels. courtship behavior, which is believed to be controlled by $\sim 2000$ neurons in the CNS (Manoli et al. 2005; Hampel et al. 2011), indicating persistent function of ectopic White protein in the CNS. Thus, the multiple roles of White are supported by temporal expression patterns from difference tissues.

CNS-located serotonin neurons provide a tissue basis for additional roles for White other than in controlling eye color in pigment cells. These neurons release serotonin (5-HT), which acts as a neuromodulator involved in locomotor behavior, circadian entrainment, and place memory (Yuan et al. 2005; Sitaraman et al. 2008; Nall and Sehgal 2014; Okusawa et al. 2014; Silva et al. 2014). Recovery from anoxia includes the restoration of 5-HT vesicular stores that would be excessively released during anoxic coma (Hansen 1985). Normal expression of White increases vesicular content of 5-HT (Borycz et al. 2008; Sitaraman et al. 2008), suggesting the presence of White in serotonin neurons. Here we show that RNAi knockdown of $w$ in dopaminergic and serotonergic neurons, or serotonin subsets, delays locomotor recovery; providing further evidence that White is expressed in the CNS and possesses nonretinal function.

The molecular basis for multiple roles of White is perhaps related to its function of nonspecific substrate transport. White, together with its transporting partners, accumulates a variety of small molecules into granules or vesicles (Anaka et al. 2008). The formation of vesicular stores preserves a sufficient amount of neurotransmitters or second messengers and improves signaling efficacy. Downregulation of White would increase the chances of exposing transmitters and other substrates to cytosolic enzymatic environments and would result in excessive hydrolysis (Evans et al. 2008). This is supported by the observation that White downregulation shifts the timing of locomotor recovery from consistently fast to remarkably slow with large variance.

\section{Insufficient rescue of delayed locomotor recovery by $m w^{+}$and its implication}

A greatly shortened $5^{\prime}$ regulatory region of $w^{+}$loses longrange transcriptional regulation (Pirrotta and Bröckl 1984). Deletion of a large intronic fragment could result in reduced skipping of downstream exon and constitutive or widespread expression (Fox-Walsh et al. 2005; Niu and Yang 2011). Additionally, $\mathrm{m} w^{+}$relocated to autosomes picks up local cisregulatory information and reduces dosage compensation (Hazelrigg 1987; Qian and Pirrotta 1995). If the $w^{+}$system were extremely optimized, the insertion of $\mathrm{m} w^{+}$would result in either no or a very limited effect on the rescue of locomotor recovery.

Although mutants of $w$ and flies with RNAi knockdown of $w$ in serotonin subsets demonstrate the same outcome (delayed locomotor recovery from anoxia) the genetic underpinnings are clearly different. Downregulation of White through an RNAi approach in restricted cell populations is sufficient to delay locomotor recovery, and downregulation in different subsets yields a uniform delay. Each of the serotonin subsets consists of $<100$ neurons in the CNS. The 
uniform delay induced by such a small population indicates a critical role for White in highly specified or differentiated neurons. This could explain the insufficiency to rescue fast locomotor recovery in w1118 flies by genetic expression of $\mathrm{m} w^{+}$. Most likely, expression levels and tissue patterns must be fully recovered to generate the wild-type phenotype. However, $\mathrm{m} w^{+}$expression is subjected to a strong position effect (Hazelrigg et al. 1984; Levis et al. 1985a; Levis et al. 1985b). Taking into account the loss of a large intronic sequence and altered dosage compensation, rescuing the locomotor recovery phenotype in w1118 mutant might not be possible by genetic manipulation of $\mathrm{m} w^{+}$.

Notwithstanding the insufficient rescue of locomotor recovery phenotype by $\mathrm{m} w^{+}$, flies carrying $w^{+}$duplicated to the Y chromosome (Brosseau et al. 1961) display wild-type-like, fast locomotor recovery from anoxia. Such a complete rescue supports the association between the $w$ gene and locomotor recovery phenotype.

Future work should focus on the molecular mechanisms underlying the $w$-associated fast locomotor recovery from anoxia. Particularly, identifying the specific transported substrates that are responsible for the timing of locomotor recovery would be valuable.

\section{Acknowledgments}

Funded by a Discovery grant from the Natural Sciences and Engineering Research Council of Canada to RMR.

\section{Literature Cited}

Agam, K., M. von Campenhausen, S. Levy, H. C. Ben-Ami, B. Cook et al., 2000 Metabolic stress reversibly activates the Drosophila light-sensitive channels TRP and TRPL in vivo. J. Neurosci. 20: 5748-5755.

Anaka, M., C. D. MacDonald, E. Barkova, K. Simon, R. Rostom et al., 2008 The white gene of Drosophila melanogaster encodes a protein with a role in courtship behavior. J. Neurogenet. 22: $243-276$.

Armstrong, G. A., C. Xiao, J. L. Krill, L. Seroude, K. Dawson-Scully et al., 2011 Glial Hsp70 protects $\mathrm{K}+$ homeostasis in the Drosophila brain during repetitive anoxic depolarization. PLoS One 6: e28994.

Bingham, P. M., and B. H. Judd, 1981 A copy of the copia transposable element is very tightly linked to the Wa allele at the white locus of D. melanogaster. Cell 25: 705-711.

Borycz, J., J. A. Borycz, A. Kubów, V. Lloyd, and I. A. Meinertzhagen, 2008 Drosophila ABC transporter mutants white, brown and scarlet have altered contents and distribution of biogenic amines in the brain. J. Exp. Biol. 211: 3454-3466.

Brand, A. H., and N. Perrimon, 1993 Targeted gene expression as a means of altering cell fates and generating dominant phenotypes. Development 118: 401-415.

Brosseau, G. E., B. Nicoletti, E. H. Grell, and D. L. Lindsley, 1961 Production of altered Y chromosomes bearing specific sections of the $\mathrm{X}$ chromosome in Drosophila. Genetics 46: 339-346.

Campbell, J. L., and H. A. Nash, 2001 Volatile general anesthetics reveal a neurobiological role for the white and brown genes of Drosophila melanogaster. J. Neurobiol. 49: 339-349.
Dimitroff, B., K. Howe, A. Watson, B. Campion, H. G. Lee et al., 2012 Diet and energy-sensing inputs affect TorC1-mediated axon misrouting but not TorC2-directed synapse growth in a Drosophila model of tuberous sclerosis. PLoS One 7: e30722.

Dreesen, T. D., D. H. Johnson, and S. Henikoff, 1988 The brown protein of Drosophila melanogaster is similar to the white protein and to components of active transport complexes. Mol. Cell. Biol. 8: 5206-5215.

Evans, J. M., J. P. Day, P. Cabrero, J. A. Dow, and S. A. Davies, 2008 A new role for a classical gene: white transports cyclic GMP. J. Exp. Biol. 211: 890-899.

Ferré, J., F. J. Silva, M. D. Real, and J. L. Ménsua, 1986 Pigment patterns in mutants affecting the biosynthesis of pteridines and xanthommatin in Drosophila melanogaster. Biochem. Genet. 24: $545-569$.

Fox-Walsh, K. L., Y. Dou, B. J. Lam, S. P. Hung, P. F. Baldi et al., 2005 The architecture of pre-mRNAs affects mechanisms of splice-site pairing. Proc. Natl. Acad. Sci. USA 102: 1617616181.

Garfinkel, M. D., V. E. Sollars, X. Lu, and D. M. Ruden, 2004 Multigenerational selection and detection of altered histone acetylation and methylation patterns: toward a quantitative epigenetics in Drosophila. Methods Mol. Biol. 287: 151-168.

Gehring, W. J., and R. Paro, 1980 Isolation of a hybrid plasmid with homologous sequences to a transposing element of Drosophila melanogaster. Cell 19: 897-904.

Ghosh, D., and H. S. Forrest, 1967 Enzymatic studies on the hydroxylation of kynurenine in Drosophila melanogaster. Genetics 55: 423-431.

Giang, T., Y. Ritze, S. Rauchfuss, M. Ogueta, and H. Scholz, 2011 The serotonin transporter expression in Drosophila melanogaster. J. Neurogenet. 25: 17-26.

Green, M. M., 1949 A Study of Tryptophane in Eye Color Mutants of Drosophila. Genetics 34: 564-572.

Greenspan, R. J., 2004 Fly pushing: the theory and practice of Drosophila genetics, Cold Spring Harbor Laboratory Press, Cold Spring Harbor, NY.

Hampel, S., P. Chung, C. E. McKellar, D. Hall, L. L. Looger et al., 2011 Drosophila Brainbow: a recombinase-based fluorescence labeling technique to subdivide neural expression patterns. Nat. Methods 8: 253-259.

Hansen, A. J., 1985 Effect of anoxia on ion distribution in the brain. Physiol. Rev. 65: 101-148.

Haywood, A. F., and B. E. Staveley, 2004 Parkin counteracts symptoms in a Drosophila model of Parkinson's disease. BMC Neurosci. 5: 14.

Hazelrigg, T., 1987 The Drosophila white gene: a molecular update. Trends Genet. 3: 43-47.

Hazelrigg, T., R. Levis, and G. M. Rubin, 1984 Transformation of white locus DNA in drosophila: dosage compensation, zeste interaction, and position effects. Cell 36: 469-481.

Hing, A. L., and J. R. Carlson, 1996 Male-male courtship behavior induced by ectopic expression of the Drosophila white gene: role of sensory function and age. J. Neurobiol. 30: 454-464.

Huser, A., A. Rohwedder, A. A. Apostolopoulou, A. Widmann, J. E. Pfitzenmaier et al., 2012 The serotonergic central nervous system of the Drosophila larva: anatomy and behavioral function. PLoS One 7: e47518.

Jenett, A., G. M. Rubin, T. T. Ngo, D. Shepherd, C. Murphy et al., 2012 A GAL4-driver line resource for Drosophila neurobiology. Cell Reports 2: 991-1001.

Kain, J. S., C. Stokes, and B. L. de Bivort, 2012 Phototactic personality in fruit flies and its suppression by serotonin and white. Proc. Natl. Acad. Sci. USA 109: 19834-19839.

Kramer, J. M., and B. E. Staveley, 2003 GAL4 causes developmental defects and apoptosis when expressed in the developing eye of Drosophila melanogaster. Genet. Mol. Res. 2: 43-47. 
Krishnan, S. N., Y. Sun, A. Mohsenin, R. J. Wyman, and G. G. Haddad, 1997 Behavioral and Electrophysiologic Responses of Drosophila melanogaster to Prolonged Periods of Anoxia. J. Insect Physiol. 43: 203-210.

Krstic, D., W. Boll, and M. Noll, 2013 Influence of the white locus on the courtship behavior of Drosophila males. PLoS One 8: e77904.

Levis, R., T. Hazelrigg, and G. M. Rubin, 1985a Effects of genomic position on the expression of transduced copies of the white gene of Drosophila. Science 229: 558-561.

Levis, R., T. Hazelrigg, and G. M. Rubin, 1985b Separable cisacting control elements for expression of the white gene of Drosophila. EMBO J. 4: 3489-3499.

Liu, L., R. L. Davis, and G. Roman, 2007 Exploratory activity in Drosophila requires the kurtz nonvisual arrestin. Genetics 175: 1197-1212.

Mackenzie, S. M., M. R. Brooker, T. R. Gill, G. B. Cox, A. J. Howells et al., 1999 Mutations in the white gene of Drosophila melanogaster affecting $\mathrm{ABC}$ transporters that determine eye colouration. Biochim. Biophys. Acta 1419: 173-185.

Mackenzie, S. M., A. J. Howells, G. B. Cox, and G. D. Ewart, 2000 Sub-cellular localisation of the white/scarlet ABC transporter to pigment granule membranes within the compound eye of Drosophila melanogaster. Genetica 108: 239-252.

Manoli, D. S., M. Foss, A. Villella, B. J. Taylor, J. C. Hall et al., 2005 Male-specific fruitless specifies the neural substrates of Drosophila courtship behaviour. Nature 436: 395-400.

Morgan, T. H., 1910 Sex limited inheritance in Drosophila. Science 32: $120-122$.

Nall, A., and A. Sehgal, 2014 Monoamines and sleep in Drosophila. Behav. Neurosci. 128: 264-272.

Niu, D. K., and Y. F. Yang, 2011 Why eukaryotic cells use introns to enhance gene expression: splicing reduces transcription-associated mutagenesis by inhibiting topoisomerase I cutting activity. Biol. Direct 6: 24.

Nolte, D. J., 1950 The eye-pigmentary system of Drosophila: The pigment cells. J. Genet. 50: 79-99.

Nolte, D. J., 1954 The eye-pigmentary system of Drosophila: The pigments of the vermilion group of mutants. J. Genet. 52: 111126.

O'Hare, K., C. Murphy, R. Levis, and G. M. Rubin, 1984 DNA sequence of the white locus of Drosophila melanogaster. J. Mol. Biol. 180: 437-455.

Okusawa, S., H. Kohsaka, and A. Nose, 2014 Serotonin and downstream leucokinin neurons modulate larval turning behavior in Drosophila. J. Neurosci. 34: 2544-2558.

Pfeiffer, B. D., A. Jenett, A. S. Hammonds, T. T. Ngo, S. Misra et al., 2008 Tools for neuroanatomy and neurogenetics in Drosophila. Proc. Natl. Acad. Sci. USA 105: 9715-9720.

Pfeiffer, B. D., T. T. Ngo, K. L. Hibbard, C. Murphy, A. Jenett et al., 2010 Refinement of tools for targeted gene expression in Drosophila. Genetics 186: 735-755.

Pirrotta, V., 1988 Vectors for P-mediated transformation in Drosophila, pp. 437-456 in Vectors: a survey of molecular cloning vectors and their uses, edited by R. I. Rodriguez, and D. T. Denhardt. Butterworth-Heinemann, Boston.

Pirrotta, V., and C. Bröckl, 1984 Transcription of the Drosophila white locus and some of its mutants. EMBO J. 3: 563-568.

Qian, S., and V. Pirrotta, 1995 Dosage compensation of the Drosophila white gene requires both the $\mathrm{X}$ chromosome environment and multiple intragenic elements. Genetics 139: 733-744.

Rezával, C., S. Werbajh, and M. F. Ceriani, 2007 Neuronal death in Drosophila triggered by GAL4 accumulation. Eur. J. Neurosci. 25: 683-694.
Sepp, K. J., J. Schulte, and V. J. Auld, 2001 Peripheral glia direct axon guidance across the CNS/PNS transition zone. Dev. Biol. 238: 47-63.

Silva, B., N. I. Goles, R. Varas, and J. M. Campusano, 2014 Serotonin receptors expressed in Drosophila mushroom bodies differentially modulate larval locomotion. PLoS One 9: e89641.

Simon, A. F., R. Daniels, R. Romero-Calderón, A. Grygoruk, H. Y. Chang et al., 2009 Drosophila vesicular monoamine transporter mutants can adapt to reduced or eliminated vesicular stores of dopamine and serotonin. Genetics 181: 525-541.

Sitaraman, D., M. Zars, H. Laferriere, Y. C. Chen, A. Sable-Smith et al., 2008 Serotonin is necessary for place memory in Drosophila. Proc. Natl. Acad. Sci. USA 105: 5579-5584.

Stoleru, D., Y. Peng, J. Agosto, and M. Rosbash, 2004 Coupled oscillators control morning and evening locomotor behaviour of Drosophila. Nature 431: 862-868.

Sullivan, D. T., and M. C. Sullivan, 1975 Transport defects as the physiological basis for eye color mutants of Drosophila melanogaster. Biochem. Genet. 13: 603-613.

Sullivan, D. T., L. A. Bell, D. R. Paton, and M. C. Sullivan, 1979 Purine transport by malpighian tubules of pteridinedeficient eye color mutants of Drosophila melanogaster. Biochem. Genet. 17: 565-573.

Sullivan, D. T., L. A. Bell, D. R. Paton, and M. C. Sullivan, 1980 Genetic and functional analysis of tryptophan transport in Malpighian tubules of Drosophila. Biochem. Genet. 18: 1109-1130.

Takano, T., G. F. Tian, W. Peng, N. Lou, D. Lovatt et al., 2007 Cortical spreading depression causes and coincides with tissue hypoxia. Nat. Neurosci. 10: 754-762.

Tearle, R. G., J. M. Belote, M. McKeown, B. S. Baker, and A. J. Howells, 1989 Cloning and characterization of the scarlet gene of Drosophila melanogaster. Genetics 122: 595-606.

Vallés, A. M., and K. White, 1988 Serotonin-containing neurons in Drosophila melanogaster: development and distribution. J. Comp. Neurol. 268: 414-428.

Waaler, G. H. M., 1921 The location of a new second chromosome eye colour gene in Drosophila melanogaster. Hereditas 2: 391-394.

Wang, H. D., P. Kazemi-Esfarjani, and S. Benzer, 2004 Multiplestress analysis for isolation of Drosophila longevity genes. Proc. Natl. Acad. Sci. USA 101: 12610-12615.

Ward, L., 1923 The genetics of curly wing in Drosophila. Another case of balanced lethal factors. Genetics 8: 276-300.

Wu, J. S., and L. Luo, 2006 A protocol for dissecting Drosophila melanogaster brains for live imaging or immunostaining. Nat. Protoc. 1: 2110-2115.

Xiao, C., V. Mileva-Seitz, L. Seroude, and R. M. Robertson, 2007 Targeting HSP70 to motoneurons protects locomotor activity from hyperthermia in Drosophila. Dev. Neurobiol. 67: 438-455.

Xiao, C., and R. M. Robertson, 2015 Locomotion induced by spatial restriction in adult Drosophila. PLoS One 10: e0135825.

Yuan, Q., F. Lin, X. Zheng, and A. Sehgal, 2005 Serotonin modulates circadian entrainment in Drosophila. Neuron 47: 115127.

Zhang, S., R. Binari, R. Zhou, and N. Perrimon, 2010 A genomewide RNA interference screen for modifiers of aggregates formation by mutant Huntingtin in Drosophila. Genetics 184: $1165-1179$.

Zhang, S. D., and W. F. Odenwald, 1995 Misexpression of the white (w) gene triggers male-male courtship in Drosophila. Proc. Natl. Acad. Sci. USA 92: 5525-5529.

Communicating editor: M. F. Wolfner 